\title{
Rosacea Treatment: Review and Update
}

Hanlin Zhang $\cdot$ Keyun Tang $\cdot$ Yuchen Wang $\cdot$ Rouyu Fang •

Qiuning Sun (10)

Received: August 21, 2020 / Published online: November 10, 2020

(C) The Author(s) 2020

\section{ABSTRACT}

Rosacea is a chronic and inflammatory skin disease characterized by flushing, nontransient erythema, papules/pustules, telangiectasia, and phymatous changes. Secondary manifestations, such as itching, burning, or stinging, are often observed in patients with rosacea. In 2017, a phenotype-based approach for diagnosis and classification was recommended. With the update of the diagnosis and classification of rosacea, treatment options for patients with rosacea have attracted the attention of dermatologists. Here, we summarize the latest advances in rosacea treatment, including skin care and cosmetic treatments, topical therapies, oral therapies, laser- and light-based therapies, injection therapies, treatments for specific types of rosacea, treatments for systemic comorbidities, and combination therapies. The impact of the phenotype-based approach on rosacea treatment and future directions are also discussed.

Keywords: Oral therapies; Rosacea; Systemic comorbidities; Treatment; Topical therapies

H. Zhang · K. Tang · Y. Wang · R. Fang · Q. Sun ( $₫)$ Department of Dermatology, Peking Union Medical College Hospital, Peking Union Medical College, Chinese Academy of Medical Sciences, Beijing 100730, China

e-mail: doctorjenny1@163.com

\section{Key Summary Points}

Rosacea is a chronic and inflammatory skin disease, characterized by flushing, nontransient erythema, papules/pustules, telangiectasia, and phymatous changes.

With the update of diagnosis and classification of rosacea in 2017, treatment options for rosacea patients also attracted the attention of dermatologists.

The latest advances in rosacea treatment include skin care and cosmetic treatments, topical therapies, oral therapies, laser- and light-based therapies, injection therapies, treatments for specific types of rosacea, treatments for systemic comorbidities, and combination therapies.

This review may act as a supplement of recent rosacea treatment guidelines and expert consensus.

Large-scale clinical research based on the phenotype approach is highly warranted in the future. 


\section{DIGITAL FEATURES}

This article is published with digital features to facilitate understanding of the article. You can access the digital features on the article's associated Figshare page. To view digital features for this article go to https://doi.org/10.6084/m9. figshare.13116755.

\section{INTRODUCTION}

Rosacea is a chronic and inflammatory disease with a series of facial skin manifestations, including flushing, nontransient erythema, papules/pustules, telangiectasia, and phymatous changes [1-4]. Secondary manifestations, such as itching, burning, or stinging, are often observed in patients with rosacea [5]. The pathogenesis and pathophysiology of rosacea are not fully understood [6-8], but immune dysfunction, Demodex infection, and exposure to ultraviolet radiation are considered to be contributing factors [2]. In 2002, rosacea was classified into the following four subtypes: erythematotelangiectatic, papulopustular, phymatous, and ocular [9]. In 2017, a phenotype-based approach for diagnosis and classification was recommended [2, 10-12]. Fixed centrofacial erythema and phymatous changes are independently considered as diagnostic criteria for rosacea [10]. In recent years, a variety of treatment options have been provided in rosacea treatment guidelines and expert consensuses [13-16], including topical therapies, oral therapies, light devices, skin care, and lifestyle management [13]. High-quality clinical trials have been conducted in recent years on rosacea treatment $[17,18]$. A systematic review, including GRADE assessments on interventions for rosacea, has been especially helpful for clinical decision-making $[19,20]$. However, treatment options for rosacea should also be expanded because managing rosacea remains a challenge [21]. The focus of this review is the expansion of treatment options rather than the level of evidence and the degree of recommendations, with the aim to summarize and update treatment options for rosacea and to supplement recent guidelines and expert consensuses.
This article is based on previously conducted studies and does not contain any new studies with human participants or animals performed by any of the authors.

\section{SKIN CARE AND COSMETIC TREATMENTS}

Patients with rosacea are encouraged to practise gentle skin care, with a focus on moderation in cleansing and moisturizing and the use of sun screens, due to the impairment of the epidermal barrier function as well as the sensitive and easily irritated nature of their facial skin [13, 22-24]. A recent study of 999 rosacea cases and 1010 controls revealed that a high frequency of cleansing and expansive use of cleansers were positively correlated with rosacea occurrence [22]. Rosacea occurs on the face, and it has a waxing and waning course, and negative impacts on emotional and overall well-being [5]. Dermatologists should educate patients with rosacea on the characteristics of the disease, the need to avoid triggers, and the importance of skin care and lifestyle management [5].

In 2018, the Society for Dermopharmacy published a guideline on dermocosmetics for use in rosacea [25]. The efficacy of and tolerance to these cosmetics should be demonstrated. The product documentation should be available to dermatologists and pharmacists. Guertler and colleagues evaluated the efficacy and safety of micellar water, cream, and serum in 50 Caucasian patients with rosacea and observed a significant reduction of facial erythema and rosacea-associated symptoms [26]. Baldwin and colleagues assessed the efficacy and tolerability of a tinted daily SPF-30 facial moisturizer on rosacea-prone skin in 33 females with mild to moderate rosacea and nontransient erythema in terms of coverage of skin redness and feelings of skin dryness and tightness/tension, reporting that the moisturizer did improve the skin appearance of rosacea-prone patients [27]. A study including 42 subjects demonstrated that a regimen consisting of a wash foam, daily sunscreen cream, and night cream improved skin hydration and skin barrier function [28], with $>$ 
$90 \%$ of the subjects reporting that the regimen was pleasant and effective and that they would continue to use this regimen and buy the products.

\section{TOPICAL THERAPIES}

In a recent systematic review on rosacea interventions based on a phenotype approach, topical brimonidine (high-certainty evidence) and topical oxymetazoline (moderate-certainty evidence) were recommended for temporarily persistent erythema [19]. Topical azelaic acid (high-certainty evidence), topical ivermectin (high-certainty evidence), topical metronidazole (moderate-certainty evidence), and topical minocycline (moderate-certainty evidence) were recommended for the treatment of papules/pustules [19]. In a systematic review and meta-analysis, topical ivermectin was demonstrated to be the most effective topical treatment for papulopustular rosacea and to provide the greatest psychological benefit to these patients [29]. Topical ivermectin can be applied not only to skin almost clear of papulopustular rosacea or with mild rosacea, but also to skin with moderate to severe rosacea [4].

Minocycline foam is a novel drug that was first used to treat moderate to severe acne vulgaris [30]. The efficacy and safety of minocycline foam for the treatment of moderate to severe papulopustular rosacea were evaluated and confirmed in two phase 3 , randomized clinical trials $(751$ and 771 participants, respectively) [18]. A prospective, 12-week, double-blinded study including 270 patients showed that 3\% topical minocycline gel benefitted patients with papulopustular rosacea, significantly decreasing inflammatory lesions compared to vehicle [31]. A higher proportion of patients using minocycline gel achieved success according to an Investigator's Global Assessment.

A significant association exists between $D e$ modex infestation and rosacea [32, 33]. High mean Demodex densities are observed in rosacea with papulopustules [34]. The impact of topical benzyl benzoate (+ crotamiton) treatment on Demodex densities and clinical symptoms of rosacea and demodicosis was assessed in a retrospective study with 394 patients (117 with rosacea and 277 with demodicosis) [35], with similar results observed in both patient cohorts. The treatment was effective in $46 \%$ of the patients and curative in $20 \%$ of the patients, and higher dose regimens were more effective than lower dose regimens. In an additional study including 344 patients, Demodex densities normalized with topical benzyl benzoate (+ crotamiton) treatment, with the symptoms clearing up in $>80 \%$ of patients with good compliance; however, the treatment was less effective and slower in poorly compliant patients [34]. The higher dose was associated with better compliance and faster results, suggesting that the higher dose of topical benzyl benzoate (+ crotamiton) might be useful for patients with rosacea and demodicosis.

Topical or systemic tranexamic acid is a conventional treatment for melasma $[36,37]$. Recent research has expanded the use of tranexamic acid in rosacea treatment. Tranexamic acid may improve the symptoms of patients with rosacea by regulating the immune response and angiogenesis [38]. In 2013, Kim and colleagues treated six Korean women diagnosed with papulopustular rosacea or rosacea with irritant contact dermatitis using tranexamic acid solution soaking [39]. Marked decreases in the quartile scores for erythema were observed in all cases. In addition, there were notable decreases in the visual analog scale scores for itching, flushing, and burning, and no significant adverse events were observed [39]. Topical tranexamic acid was also used to treat erythematotelangiectatic rosacea in two other studies (with 20 patients and 1 patient, respectively), with the results of both studies implying promising therapeutic effects with a good safety profile $[40,41]$.

\section{ORAL THERAPIES}

A systemic treatment or combination therapy should be considered for managing moderate to severe papulopustular rosacea [15]. The US Food and Drug Administration has approved modified-release oral doxycycline capsules for the 
treatment of inflammatory papules/pustules of rosacea [13]. Doxycycline $40 \mathrm{mg}$ modified release (moderate-to-high-certainty evidence), isotretinoin (moderate-to-high-certainty evidence), and minocycline (moderate-certainty evidence) have been recommended as treatment for reducing papules/pustules in the updated systematic review on interventions for rosacea [19].

Oral $\beta$-blockers might be useful to treat persistent erythema and flushing because they antagonize the effects of sympathetic nerve stimulation and circulating catecholamines at $\beta$-adrenoceptors [42-44]. In a systematic review including nine studies, Logger and colleagues concluded that oral nonselective $\beta$-blockers, especially carvedilol and propranolol, may be an effective treatment option for rosacea-associated facial flushing and erythema [43]. In a monocentric retrospective study, five Caucasian rosacea patients received carvedilol treatment titrated up to $12.5 \mathrm{mg}$ twice daily for at least 6 months [45]. All patients had a remarkable decrease in the self-assessment and clinician's erythema assessment grades, and they reported a major improvement of satisfaction. Large, prospective, clinical trials are needed to confirm the effect of $\beta$-blockers in rosacea treatment. The optimal dosage and long-term effects also need to be determined.

Hydroxychloroquine, which is used in patients with systemic autoimmune diseases, is a new treatment option for rosacea patients $[46,47]$. In a rosacea-like mouse model, hydroxychloroquine was shown to ameliorate rosacea-like dermatitis, possibly by regulating the immune response of mast cells [46]. A randomized, double-blind, double-dummy study of 66 patients showed that the difference in changes in total rosacea-specific quality of life scores in the hydroxychloroquine group was noninferior to that in the doxycycline group. However, no noninferior results were observed in the clinician's erythema assessment success rate, the investigator's global assessment success of "clear" or "almost clear" rate, and excellent improvement rate [47]. Dry skin (14.3\%), dry eye $(7.1 \%)$, and dizziness $(7.1 \%)$ were the most common adverse events in the hydroxychloroquine group. Hydroxychloroquine has the advantage of being a generally safe medication during pregnancy; however, more studies with larger sample size are warranted to demonstrate the effectiveness and safety of hydroxychloroquine.

\section{LASER- AND LIGHT-BASED THERAPIES}

Laser and intense pulsed light (IPL) therapy (low-to-moderate-certainty evidence) are recommended for the treatment of erythema and mainly telangiectasia in the updated systematic review on interventions for rosacea [19]. No significant difference in either the melanin or erythema index was observed between shortpulsed ILP therapy and pulsed dye laser therapy for rosacea treatment when used at the same energies and pulse [48]. In a retrospective study of 807 patients with different subtypes of rosacea, including erythema/telangiectasia, papules/pustules, and rhinophyma, Zhang and colleagues also noted that the multiple, sequential light/laser devices may be used to treat nasal rosacea [49]. In a study including 40 patients, treatment with a 577-nm pro-yellow laser was also demonstrated to be an effective and safe treatment for erythematotelangiectatic rosacea, facial erythema, and facial telangiectasia [50].

In a previous case report, a patient with recalcitrant granulomatous rosacea was treated with $\delta$-aminolaevulinic acid-photodynamic therapy (ALA-PDT) $[15,51]$, but six sessions were required. Two studies have also shown that ALA-PDT is an effective and safe treatment for erythematotelangiectatic or papulopustular rosacea $[52,53]$; however, the sample size in these studies was small, with only 17 and 20 patients enrolled, respectively. Of note, the selection of proper parameters is required in studies involving laser- and light-based therapies because flares of rosacea may be triggered at high levels of photodynamic therapy [53].

The combination of laser- and light-based therapies and other therapies has also been investigated in recent studies. The findings of a phase 4 , multicenter, interventional, open-label study with 46 patients suggest the benefit of 
oxymetazoline as adjunctive therapy with energy-based therapy for the treatment of moderate to severe persistent facial erythema associated with rosacea [54]. A retrospective study including 31 subjects revealed that the combination of pulsed dye laser and 1.0\% oxymetazoline cream reduced erythema and telangiectasias both safely and effectively [55]. An open-label study including 21 subjects assessed the efficacy and tolerability of topical skin care and sun protection combined with IPL therapy [56]. After using a topical skin care regimen (TSCR) monotherapy for 12 weeks, subjects received a single IPL light therapy, following which they continued the TSCR monotherapy for another 6 weeks. When used as a monotherapy, TSCR decreased investigatorrated erythema, but the improvement did not achieve significance. All subjects agreed that TSCR improved their skin redness, and $80 \%$ of the subjects felt satisfied or very satisfied with the TSCR at week 18.

\section{INJECTION THERAPIES}

To date, two injection therapies have been demonstrated to be useful in rosacea treatment, namely, botulinum toxin and interleukin (IL)17 inhibitors. In a double-blinded, placebocontrolled, split-face study, 24 participants were randomized to the intervention and placebo groups and given intradermal injections of botulinum toxin and normal saline in both cheeks, respectively [57]. A significant decrease in the erythema index and improved skin elasticity were observed in the group receiving the botulinum toxin injection. A case report also showed that intradermal injections of botulinum toxin significantly reduced erythema, edema, telangiectasias, and flushing [58]. In another study, 20 rosacea patients received the combination therapy of pulsed dye laser and intradermal botulinum toxin [59]. A synergistic effect was observed in this combination therapy, which showed high efficacy, received a high satisfaction rating by patients, and had a low side-effect profile. However, the cost and the need for repeated injections must also be taken into consideration.
Another injection therapy recently used in rosacea treatment are IL-17 inhibitors. Ali and colleagues reviewed the role of IL-17 in papulopustular rosacea [60] and found that IL-17 links Demodex folliculorum to angiogenesis, telangiectasias, inflammation, and pustules. Although treatment with IL-17 inhibitors are costly, these agents might be used to treat severe and treatment-resistant papulopustular rosacea. The authors of an exploratory, openlabel, investigator-initiated study of 20 patients receiving secukinumab (300 $\mathrm{mg}$ weekly for 5 weeks and then monthly for 2 months) reported a significant reduction in papules and global severity score [61]. However, 39\% of the patients in this study receiving at least one dose experienced at least one infection. Larger, randomized controlled studies that included comparison groups are necessary to evaluate the efficacy and safety of IL-17 inhibitors.

\section{SPECIFIC TYPES OF ROSACEA}

\section{Ocular Rosacea}

Ocular involvement may be observed in more than $50 \%$ of rosacea patients [13]. An interdisciplinary review noted that eyelid hygiene and systemic treatment with tetracycline drugs should be used as a treatment for ocular rosacea [62]. Oral omega-3 fatty acids (moderate-certainty evidence), ciclosporin ophthalmic emulsion (low-certainty evidence), and doxycycline (low-certainty evidence) are recommended for the treatment of ocular rosacea [19]. Based on their clinical experience, Vazirnia and colleagues noted that IPL might also improve dry eye symptoms in patients with rosacea following treatment of periocular facial skin [63]. These authors also noted that eye shields should be properly applied for eye protection. In another study, three patients with serious ocular rosacea also benefited from an improved water-soluble silver(I) complex of metronidazole, with improved uncorrected visual acuity test results as well as an improved objective and subjective evaluation of the tear film parameters [64]. 
Donmez and colleagues summarized the clinical characteristics of 16 patients with pediatric ocular acne rosacea, recommending flexible treatment with warm compresses, eyelid scrubbing, preservative-free artificial tears, topical antibiotics, topical steroids, topical cyclosporine, oral doxycycline, azithromycin suspension, and erythromycin suspension [65]. Early identification and treatment should be emphasized when children present with ocular irritation, meibomian gland disease, recurrent chalazia, and peripheral corneal infiltrates that are of long duration, even without skin changes [65].

\section{Phymatous Rosacea}

Phymatous rosacea was classified as a specific subtype of rosacea in the 2002 classification published by the National Rosacea Society Expert Committee on the Classification and Staging of Rosacea [9]. Phymatous rosacea mainly occurs in the nose, known as rhinophyma. In 2017, phymatous changes were designated as one of the diagnostic phenotypes of rosacea $[2,10]$. The recent rosacea treatment update from the global ROSacea COnsensus (ROSCO) panel [66] recommends that treatment for phyma should depend on whether it is inflamed or noninflamed. For inflamed phyma or active phyma, oral doxycycline and oral isotretinoin were recommended; for noninflamed phyma or fibrotic phyma, physical modalities were recommended. Graves and colleagues used $\mathrm{CO}_{2}$ laser ablation in a patient with rhinophyma due to its efficacy, reliability, practicality, and predictability [67]. In a retrospective study of 28 patients, a porcine extracellular matrix was recommended for use after shave excision of rhinophyma; this treatment was found to reduce the number of dressing changes and time to reepithelization [68].

\section{Rosacea Fulminans}

Rosacea fulminans, also known as pyoderma faciale or rosacea conglobate, is a rare disease characterized by the erupt onset of inflammatory papules, pustules, nodules, and cysts on the face [69]. Rosacea fulminans might be associated with inflammatory bowel disease [70] and pregnancy [71]. The authors of a comprehensive review on the diagnosis and treatment of rosacea fulminans recommended systemic treatment with corticosteroid and/or isotretinoin, which may improve symptoms and reduce rates of scarring [69].

\section{Granulomatous Rosacea}

Granulomatous rosacea was also recognized as a variant of rosacea in 2002 by the National Rosacea Society Expert Committee on the Classification and Staging of Rosacea [9]. However, in a more recent classification system, granulomatous rosacea was not mentioned $[2,10]$. This disease often presents a chronic course, which is challenging to manage [72]. Only case reports and case series have been published on the treatment of granulomatous rosacea treatment [15]. Successful treatment with chromophore gel-assisted phototherapy [73], topical ivermectin [74], topical brimonidine [75], oral doxycycline [75], oral metronidazole [74], oral steroids [75], and oral dapsone [72] has been documented in recent years in these case reports.

\section{SYSTEMIC COMORBIDITIES}

Rosacea has been associated with a series of cardiovascular diseases, gastrointestinal disorders, neurologic disorders, and psychiatric disorders $[12,76,77]$. Wollina noted that rosacea was associated with metabolic, psychiatric, and neurologic disorders and specific types of cancer, and Wollina suggested that rosacea should be considered a systemic disease [78].

Chronic inflammation in rosacea patients may be systemic, and inflammation may link rosacea and cardiometabolic disease [79]. Skin microbiota may also act as a link between rosacea and its comorbidities [80]. Recognizing and screening systemic disorders in rosacea patients may be beneficial in the early diagnosis and treatment of these diseases [79, 81]. To date, however, no direct treatment options have focused on the comorbidities of rosacea. In 
2018, Kallis and colleagues reviewed a biologically based approach to treat acne and rosacea [82]. Complementary and alternative medicine therapies have been used to treat acne and rosacea due to the chronicity of these dermatologic conditions. These publicly approved holistic approaches suggest that rosacea is a systemic disease [82]. Studies on comorbidities that contribute to or arise from rosacea are needed in further research [11].

\section{COMBINATION THERAPIES}

Combination therapy to target specific features is necessary to achieve effective treatment, especially in patients with severe rosacea or multiple rosacea features $[13,14]$. Oral therapy combined with topical therapy [83], laser-/lightbased therapy combined with topical therapy [54], and a combination of topical therapies [84] have all been documented in recent publications. Fractional microneedling radiofrequency, a new device in dermatology, has also been used to treat recalcitrant papulopustular rosacea combined with pulsed dye laser and oral isotretinoin [85]. This novel combination regimen showed satisfactory efficacy and acceptable safety profiles in the 25 patients enrolled in the study.

In a large cohort analysis of 72,173 patients diagnosed with rosacea, Lev-Tov and colleagues found that more than $20 \%$ of rosacea patients treated with topical agents $(n=62,074)$ received combination topical therapy [86]. However, the combined medications have similar working mechanisms and have not been studied together. Thus, the remaining question is whether to combine or not to combine the medications. More studies, especially cost-effectiveness studies, are needed to identify efficient and cost-efficient treatments for rosacea patients [86].

\section{DISCUSSION}

Rosacea treatment remains challenging for dermatologists, especially in refractory or recalcitrant cases. The pathogenesis of rosacea is not well understood. Immune dysfunction [87],
Demodex [88], ultraviolet radiation exposure [89], and vascular hyperreactivity [90] may all play a role in the pathogenesis of rosacea. The pathogenesis and related mechanisms need further exploration to develop more etiological treatment modalities. The waxing and waning course of rosacea may disturb patients to a large extent, and rosacea is also related to many psychiatric disorders, such as anxiety and depression [91]. Dermatologists should focus on patient experience and patient education, and they should schedule regular follow-ups if necessary.

The evolvement of rosacea classification from subtyping to phenotyping may make the selection of treatment more rational by emphasizing the specific presentation and concerns of the individual patient [2]. This evolvement is also in accordance with the "patientcentric approach" [92-94]. Individualized treatment should be applied to rosacea patients [95]. In the era of the phenotype approach, van Zuuren and colleagues systematically reviewed interventions for rosacea based on the new approach, using the GRADE guidelines to assess certainty of evidence [19]. Based on the systematic review published in 2019, we completed the descriptive review on treatment modalities in rosacea presented here, with special focus on the newly emerged modalities, with more emphasis on the expansion of treatment options and less emphasis on GRADE assessments. The treatment modalities that have newly emerged in the last 2 years might not be that helpful to clinical decision-making compared to those modalities recommended in that systematic review of 20,944 participants [19]. However, these emerged modalities may be applied to and be potentially useful in the treatment of refractory or recalcitrant cases. These modalities should be emphasized, and they may attract the attention of investigators who need a broader perspective and comprehensive understanding of rosacea treatment. Researchers should expand and verify rosacea treatment options, with a major focus on largescale clinical research based on the phenotype approach. 


\section{CONCLUSIONS}

In this review we summarize recent advances in rosacea treatment, including skin care and cosmetic treatments, topical therapies, oral therapies, laser-/light-based therapies, injection therapies, treatments for specific types of rosacea and treatments for systemic comorbidities, and combination therapies, in the era of phenotype-based diagnosis and classification for rosacea. There is still much to accomplish for precise treatment modalities for rosacea patients, and additional modalities also need to be developed and validated. This review may act as a supplement of recent rosacea treatment guidelines and expert consensuses. Large-scale clinical research based on the phenotype approach is highly warranted in the future.

\section{ACKNOWLEDGEMENTS}

Funding. No sponsorship was received for this study or publication of this article. The Rapid Service Fee was funded by Peking Union Medical College Hospital.

Authorship. All the named authors meet the International Committee of Medical Journal Editors (ICMJE) criteria for authorship for this article, take responsibility for the integrity of the work as a whole, and have given their approval for this version to be published.

Disclosures. Hanlin Zhang, Keyun Tang, Yuchen Wang, Rouyu Fang, and Qiuning Sun have nothing to disclose.

Medical Writing and Editorial Assistance. We thank AJE (https://www.aje.com/) for its linguistic assistance in editing the English text of the revised manuscript. The fee was funded by the authors.

Compliance with Ethics Guidelines. This article is based on previously conducted studies and does not contain any new studies with human participants or animals performed by any of the authors.
Data Availability. Data sharing is not applicable to this article as no datasets were generated or analyzed during the current study.

Open Access. This article is licensed under a Creative Commons Attribution-NonCommercial 4.0 International License, which permits any non-commercial use, sharing, adaptation, distribution and reproduction in any medium or format, as long as you give appropriate credit to the original author(s) and the source, provide a link to the Creative Commons licence, and indicate if changes were made. The images or other third party material in this article are included in the article's Creative Commons licence, unless indicated otherwise in a credit line to the material. If material is not included in the article's Creative Commons licence and your intended use is not permitted by statutory regulation or exceeds the permitted use, you will need to obtain permission directly from the copyright holder. To view a copy of this licence, visit http://creativecommons.org/licenses/by$\mathrm{nc} / 4.0 /$.

\section{REFERENCES}

1. Wang Y, Zhang H, Fang R, Tang K, Sun Q. The top 100 most cited articles in Rosacea: a bibliometric analysis. J Eur Acad Dermatol Venereol. 2020. https://doi.org/10.1111/jdv.16305.

2. Tan J, Almeida LM, Bewley A, et al. Updating the diagnosis, classification and assessment of rosacea: recommendations from the global ROSacea COnsensus (ROSCO) panel. $\mathrm{Br} \mathrm{J}$ Dermatol. 2017;176(2):431-8.

3. Dursun R, Daye M, Durmaz K. Acne and rosacea: what's new for treatment? Dermatol Ther. 2019;32(5):e13020.

4. Trave I, Merlo G, Cozzani E, Parodi A. Real-life experience on effectiveness and tolerability of topical ivermectin in papulopustular rosacea and antiparasitic effect on Demodex mites. Dermatol Ther. 2019;32(6):e13093.

5. Johnson SM, Berg A, Barr C. Managing Rosacea in the clinic: from pathophysiology to treatment-a review of the literature. J Clin Aesth Dermatol. 2020;13(4 Suppl):S17-S22. 
6. Rainer BM, Thompson KG, Antonescu C, et al. Characterization and analysis of the skin microbiota in Rosacea: a case-control study. Am J Clin Dermatol. 2020;21(1):139-47.

7. Powell FC. Clinical practice. Rosacea. N Engl J Med. 2005;352(8):793-803.

8. Ahn CS, Huang WW. Rosacea pathogenesis. Dermatol Clin. 2018;36(2):81-6.

9. Wilkin J, Dahl M, Detmar M, et al. Standard classification of rosacea: report of the National Rosacea Society Expert Committee on the Classification and Staging of Rosacea. J Am Acad Dermatol. 2002;46(4):584-7.

10. Gallo RL, Granstein RD, Kang S, et al. Standard classification and pathophysiology of rosacea: the 2017 update by the National Rosacea Society Expert Committee. J Am Acad Dermatol. 2018;78(1): 148-55.

11. Wang YA, James WD. Update on rosacea classification and its controversies. Cutis. 2019;104(1):70-3.

12. Thyssen JP. Subtyping, phenotyping or endotyping rosacea: how can we improve disease understanding and patient care? Br J Dermatol. 2018;179(3): $551-2$.

13. Thiboutot D, Anderson R, Cook-Bolden F, et al. Standard management options for Rosacea: the 2019 update by the National Rosacea Society Expert Committee. J Am Acad Dermatol. 2020;82(6): 1501-10.

14. Schaller M, Almeida LMC, Bewley A, et al. Recommendations for rosacea diagnosis, classification and management: update from the global ROSacea COnsensus 2019 panel. Br J Dermatol. 2020;182(5): 1269-76.

15. Anzengruber F, Czernielewski J, Conrad C, et al. Swiss S1 guideline for the treatment of rosacea. J Eur Acad Dermatol Venereol. 2017;31(11):1775-91.

16. Asai Y, Tan J, Baibergenova A, et al. Canadian clinical practice guidelines for Rosacea. J Cutan Med Surg. 2016;20(5):432-45.

17. Schaller M, Kemény L, Havlickova B, et al. A randomized phase $3 \mathrm{~b} / 4$ study to evaluate concomitant use of topical ivermectin 1\% cream and doxycycline 40-mg modified-release capsules, versus topical ivermectin $1 \%$ cream and placebo in the treatment of severe rosacea. J Am Acad Dermatol. 2020;82(2):336-43.

18. Gold LS, Del Rosso JQ, Kircik L, et al. Minocycline 1. $5 \%$ foam for the topical treatment of moderate to severe papulopustular rosacea: results of 2 phase 3 , randomized, clinical trials. J Am Acad Dermatol. 2020;82(5):1166-73.

19. van Zuuren EJ, Fedorowicz Z, Tan J, et al. Interventions for rosacea based on the phenotype approach: an updated systematic review including GRADE assessments. Br J Dermatol. 2019;181(1): 65-79.

20. Le Cleach L, Cribier B. New evidence but still unmet medical needs in rosacea treatment. Br J Dermatol. 2019;181(1):11-2.

21. Hampton PJ. Expanding treatment options for rosacea. Br J Dermatol. 2020. https://doi.org/10. 1111/bjd.18985.

22. Li G, Wang B, Zhao Z, et al. Excessive cleansing: an underestimating risk factor of rosacea in Chinese population. Arch Dermatol Res. 2020. https://doi. org/10.1007/s00403-020-02095-w.

23. Diczig B, Németh I, Sárdy M, Pónyai G. Contact hypersensitivity in rosacea-a report on 143 cases. J Eur Acad Dermatol Venereol. 2018;32(9):e347-9.

24. Draelos ZD, Gunt H, Levy SB. Natural skin care products as adjunctive to prescription therapy in moderate to severe Rosacea. J Drugs Dermatol. 2019;18(2):141-6.

25. Kresken J, Kindl U, Wigger-Alberti W, Clanner-Engelshofen BM, Reinholz M. Dermocosmetics for use in Rosacea: guideline of the Society for Dermopharmacy. Skin Pharmacol Physiol. 2018;31(3): 147-54.

26. Guertler A, Jøntvedt NM, Clanner-Engelshofen BM, Cappello C, Sager A, Reinholz M. Efficacy and safety results of micellar water, cream and serum for rosacea in comparison to a control group. J Cosmet Dermatol. 2020. https://doi.org/10.1111/jocd. 13591.

27. Baldwin H, Santoro F, Lachmann N, Teissedre S. A novel moisturizer with high sun protection factor improves cutaneous barrier function and the visible appearance of rosacea-prone skin. J Cosmetic Dermatol. 2019;18(6):1686-92.

28. Santoro F, Lachmann N. An open-label, intra-individual study to evaluate a regimen of three cosmetic products combined with medical treatment of Rosacea: cutaneous tolerability and effect on hydration. Dermatol Ther. 2019;9(4):775-84.

29. Husein-ElAhmed H, Steinhoff M. Efficacy of topical ivermectin and impact on quality of life in patients with papulopustular rosacea: a systematic review and meta-analysis. Dermatol Ther. 2020;33(1): e13203. 
30. Shemer A, Shiri J, Mashiah J, Farhi R, Gupta AK. Topical minocycline foam for moderate to severe acne vulgaris: phase 2 randomized double-blind, vehicle-controlled study results. J Am Acad Dermatol. 2016;74(6):1251-2.

31. Webster G, Draelos ZD, Graber E, et al. A multicentre, randomized, double-masked, parallel group, vehicle-controlled phase IIb study to evaluate the safety and efficacy of $1 \%$ and $3 \%$ topical minocycline gel in patients with papulopustular rosacea. $\mathrm{Br}$ J Dermatol. 2020. https://doi.org/10.1111/bjd. 18857.

32. Zhao YE, Wu LP, Peng Y, Cheng H. Retrospective analysis of the association between Demodex infestation and rosacea. Arch Dermatol. 2010;146(8):896-902.

33. Jacob S, VanDaele MA, Brown JN. Treatment of Demodex-associated inflammatory skin conditions: a systematic review. Dermatol Ther. 2019;32(6): e13103.

34. Forton FMN, De Maertelaer V. Effectiveness of benzyl benzoate treatment on clinical symptoms and Demodex density over time in patients with rosacea and demodicosis: a real life retrospective follow-up study comparing low- and high-dose regimens. J Dermatol Treat. 2020:1-10.

35. Forton FMN, De Maertelaer V. Treatment of rosacea and demodicosis with benzyl benzoate: effects of different doses on Demodex density and clinical symptoms. J Eur Acad Dermatol Venereol. 2020;34(2):365-9.

36. Sheu SL. Treatment of melasma using tranexamic acid: what's known and what's next. Cutis. 2018;101(2):E7-8.

37. Bala HR, Lee S, Wong C, Pandya AG, Rodrigues M. Oral tranexamic acid for the treatment of melasma: a review. Dermatol Surg. 2018;44(6):814-25.

38. Li Y, Xie H, Deng Z, et al. Tranexamic acid ameliorates rosacea symptoms through regulating immune response and angiogenesis. Int Immunopharmacol. 2019;67:326-34.

39. Kim MS, Chang SE, Haw S, Bak H, Kim YJ, Lee MW. Tranexamic acid solution soaking is an excellent approach for rosacea patients: a preliminary observation in six patients. J Dermatol. 2013;40(1):70-1.

40. Bageorgou F, Vasalou V, Tzanetakou V, Kontochristopoulos G. The new therapeutic choice of tranexamic acid solution in treatment of erythematotelangiectatic rosacea. J Cosmetic Dermatol. 2019;18(2):563-7.
41. Jakhar D, Kaur I, Misri R. Topical 10\% tranexamic acid for erythematotelangiectatic steriod induced rosacea. J Am Acad Dermatol. 2020. https://doi.org/ 10.1016/j.jaad.2019.12.067.

42. Chen L, Tsai TF. The role of $\beta$-blockers in dermatological treatment: a review. J Eur Acad Dermatol Venereol. 2018;32(3):363-71.

43. Logger JGM, Olydam JI, Driessen RJB. Use of betablockers for rosacea-associated facial erythema and flushing: a systematic review and update on proposed mode of action. J Am Acad Dermatol. 2020. https://doi.org/10.1016/j.jaad.2020.04.129.

44. Prabha N, Chhabra N, Arora R. Beta-blockers in dermatology. Indian J Dermatol Venereol Leprol. 2017;83(3):399-407.

45. Pietschke K, Schaller M. Long-term management of distinct facial flushing and persistent erythema of rosacea by treatment with carvedilol. J Dermatol Treat. 2018;29(3):310-3.

46. Li J, Yuan X, Tang Y, et al. Hydroxychloroquine is a novel therapeutic approach for rosacea. Int Immunopharmacol. 2020;79:106178.

47. Wang B, Yuan X, Huang X, et al. Efficacy and safety of hydroxychloroquine for treatment of patients with rosacea: a multi-center, randomized, doubleblind, double-dummy, pilot study. J Amer Acad Dermatol. 2020. https://doi.org/10.1016/j.jaad. 2020.05.050.

48. Kim BY, Moon HR, Ryu HJ. Comparative efficacy of short-pulsed intense pulsed light and pulsed dye laser to treat rosacea. J Cosmetic Laser Ther. 2019;21(5):291-6.

49. Zhang Y, Jiang S, Lu Y, et al. A decade retrospective study of light/laser devices in treating nasal rosacea. J Dermatol Treat. 2020;31(1):84-90.

50. Kapicioglu Y, Sarac G, Cenk H. Treatment of erythematotelangiectatic rosacea, facial erythema, and facial telangiectasia with a 577-nm pro-yellow laser: a case series. Lasers Med Sci. 2019;34(1):93-8.

51. Baglieri F, Scuderi G. Treatment of recalcitrant granulomatous rosacea with ALA-PDT: report of a case. Indian J Dermatol Venereol Leprol. 2011;77(4):536.

52. Sun Y, Chen L, Zhang Y, Gao X, Wu Y, Chen H. Topical photodynamic therapy with 5-aminolevulinic acid in Chinese patients with Rosacea. J Cosmetic Laser Ther. 2019;21(4):196-200.

53. Fan L, Yin R, Lan T, Hamblin MR. Photodynamic therapy for rosacea in Chinese patients. Photodiagn Photodyn Ther. 2018;24:82-7. 
54. Tanghetti EA, Goldberg DJ, Dover JS, et al. Oxymetazoline and energy-based therapy in patients with Rosacea: evaluation of the safety and tolerability in an open-label, interventional study. Lasers Surg Med. 2020. https://doi.org/10.1002/lsm. 23253.

55. Suggs AK, Macri A, Richmond H, Munavalli G, Friedman PM. Treatment of erythematotelangiectatic Rosacea with pulsed-dye laser and oxymetazoline $1.0 \%$ cream: a retrospective study. Lasers Surg Med. 2020;52(1):38-43.

56. Deaver Peterson J, Katz TM. Open-label study assessing the efficacy and tolerability of topical skin care and sun protection alone and in combination with intense pulsed light therapy. J Cosmetic Dermatol. 2019;18(6):1758-64.

57. Kim MJ, Kim JH, Cheon HI, et al. Assessment of skin physiology change and safety after intradermal injections with botulinum toxin: a randomized, double-blind, placebo-controlled, split-face pilot study in rosacea patients with facial erythema. Dermatol Surg. 2019;45(9):1155-62.

58. Bharti J, Sonthalia S, Jakhar D. Mesotherapy with botulinum toxin for the treatment of refractory vascular and papulopustular rosacea. J Am Acad Dermatol. 2018. https://doi.org/10.1016/j.jaad. 2018.05.014.

59. Al-Niaimi F, Glagoleva E, Araviiskaia E. Pulsed dye laser followed by intradermal botulinum toxin type-A in the treatment of rosacea-associated erythema and flushing. Dermatol Ther. 2020. https:// doi.org/10.1111/dth.13976.

60. Amir Ali A, Vender R, Vender R. The role of IL-17 in papulopustular Rosacea and future directions. J Cutan Med Surg. 2019;23(6):635-41.

61. Kumar AM, Chiou AS, Shih YH, Li S, Chang ALS. An exploratory, open-label, investigator-initiated study of interleukin-17 blockade in patients with moderate-to-severe papulopustular rosacea. Br J Dermatol. 2020. https://doi.org/10.1111/bjd.19172.

62. Jabbehdari S, Memar OM, Caughlin B, Djalilian AR. Update on the pathogenesis and management of ocular rosacea: an interdisciplinary review. Eur J Ophthalmol. 2020. https://doi.org/10.1177/ 1120672120937252.

63. Vazirnia A, Wat $H$, Danesh MJ, Anderson RR. Intense pulsed light for improving dry eye disease in rosacea. J Am Acad Dermatol. 2019. https://doi. org/10.1016/j.jaad.2019.11.045.

64. Waszczykowska A, Żyro D, Jurowski P, Ochocki J. Effect of treatment with silver(I) complex of metronidazole on ocular rosacea: design and formulation of new silver drug with potent antimicrobial activity. J Trace Elem Med Biol. 2020;61:126531.

65. Donmez O, Akova YA. Pediatric ocular acne Rosacea: clinical features and long term follow-up of sixteen cases. Ocular Immunol Inflamm. 2019:1-9.

66. Schaller M, Almeida LM, Bewley A, et al. Rosacea treatment update: recommendations from the global ROSacea COnsensus (ROSCO) panel. Br J Dermatol. 2017;176(2):465-71.

67. Graves LL, Hoopman J, Finn R. Carbon dioxide laser resurfacing for rhinophyma: a case report and discussion of the literature. J Oral Maxillofac Surg. 2020. https://doi.org/10.1016/j.joms.2020.06.012.

68. Schmitz L, Hessam S, Scholl L, Reitenbach S, Segert $\mathrm{MH}$, Bechara FG. Wound care with a porcine extracellular matrix after surgical treatment of rhinophyma. J Cutan Med Surg. 2020;24(3):253-8.

69. Walsh RK, Endicott AA, Shinkai K. Diagnosis and treatment of Rosacea fulminans: a comprehensive review. Am J Clin Dermatol. 2018;19(1):79-86.

70. Nowak M, Barańska-Rybak W, Mehrholz D, Nowicki J. Rosacea fulminans-coincidence of the disease with inflammatory bowel disease. J Eur Acad Dermatol Venereol. 2019;33(6):e247-8.

71. Garayar Cantero M, Garabito Solovera E, Aguado García Á, Valtueña J, Ruiz Sánchez D, Manchado LP. Use of permethrin in the treatment of rosacea fulminans during pregnancy: one case report. Dermatol Ther. 2020;33(3):e13436.

72. Merlo G, Cozzani E, Russo R, Parodi A. Dapsone for unresponsive granulomatous Rosacea. Am J Ther. 2020;27(3):e304-6.

73. Liu RC, Makhija M, Wong XL, Sebaratnam DF. Treatment of granulomatous rosacea with chromophore gel-assisted phototherapy. Photodermatol Photoimmunol Photomed. 2019;35(4):280-1.

74. Ansorge C, Technau-Hafsi K. Granulomatous rosacea in a lung transplant recipient: A possible therapy option in a unique group of patients. Hautarzt. 2020;71(2):134-8. [Article in German].

75. Kok WL, Oon HH, Giam YC. A case report of granulomatous rosacea of the face. Singap Med J. 2018;59(4):228-9.

76. Haber R, El Gemayel M. Comorbidities in rosacea: a systematic review and update. J Am Acad Dermatol. 2018;78(4):786-92.e8. 
77. Searle T, Al-Niaimi F, Ali FR. Rosacea and the cardiovascular system. J Cosmetic Dermatol. 2020. https://doi.org/10.1111/jocd.13587.

78. Wollina U. Is rosacea a systemic disease? Clin Dermatol. 2019;37(6):629-35.

79. Chen Q, Shi X, Tang Y, et al. Association between Rosacea and cardiometabolic disease: a systematic review and meta-analysis. J Am Acad Dermatol. 2020. https://doi.org/10.1016/j.jaad.2020.04.113.

80. Thompson KG, Rainer BM, Kang S, Chien AL. The skin microbiota as a link between rosacea and its systemic comorbidities. Int J Dermatol. 2020;59(4): 513-4.

81. Li Y, Guo L, Hao D, Li X, Wang Y, Jiang X. Association between Rosacea and cardiovascular diseases and related risk factors: a systematic review and meta-analysis. Biomed Res Int. 2020;2020:7015249.

82. Kallis PJ, Price A, Dosal JR, Nichols AJ, Keri J. A biologically based approach to acne and Rosacea. J Drugs Dermatol. 2018;17(6):611-7.

83. Bilgin B, Karadag AS. Effects of combined oral doxycycline and topical cyclosporine treatment on ocular signs, symptoms, and tear film parameters in rosacea patients. Arquivos Brasil Oftalmol. 2018;81(6):466-70.

84. Ebneyamin E, Mansouri P, Rajabi M, Qomi M, Asgharian R, Azizian Z. The efficacy and safety of permethrin $2.5 \%$ with tea tree oil gel on rosacea treatment: a double-blind, controlled clinical trial. J Cosmetic Dermatol. 2020;19(6):1426-31.

85. Kwon HH, Jung JY, Lee WY, Bae Y, Park GH. Combined treatment of recalcitrant papulopustular rosacea involving pulsed dye laser and fractional microneedling radiofrequency with low-dose isotretinoin. J Cosmetic Dermatol. 2020;19(1):105-11.

86. Lev-Tov H, Rill JS, Liu G, Kirby JS. Trends in utilization of topical medications for treatment of rosacea in the United States (2005-2014): a cohort analysis. J Am Acad Dermatol. 2019;80(4):1135-7.
87. Kulkarni NN, Takahashi T, Sanford JA, et al. Innate immune dysfunction in rosacea promotes photosensitivity and vascular adhesion molecule expression. J Invest Dermatol. 2020;140(3):645-55.e6.

88. Altunisik N, Turkmen D, Sener S. Investigation of the relationship between inflammatory blood parameters and rosacea and demodex infestation. J Cosmetic Dermatol. 2020;19(8):2105-8.

89. Aldrich N, Gerstenblith $\mathrm{M}, \mathrm{Fu}$ P, et al. Genetic vs environmental factors that correlate with rosacea: a cohort-based survey of twins. JAMA Dermatol. 2015;151(11):1213-9.

90. Mc Aleer MA, Lacey N, Powell FC. The pathophysiology of rosacea. G Ital Dermatol Venereol. 2009;144(6):663-71.

91. Incel Uysal P, Akdogan N, Hayran Y, Oktem A, Yalcin B. Rosacea associated with increased risk of generalized anxiety disorder: a case-control study of prevalence and risk of anxiety in patients with rosacea. An Bras Dermatol. 2019;94(6):704-9.

92. Elewski B. Rosacea treatment: a patient-centric approach. Br J Dermatol. 2020;182(5):1090-1.

93. Cices A, Alexis AF. Patient-focused solutions in Rosacea management: treatment challenges in special patient groups. J Drugs Dermatol. 2019;18(7):608-12.

94. Tan J, Berg M, Gallo RL, Del Rosso JQ. Applying the phenotype approach for rosacea to practice and research. Br J Dermatol. 2018;179(3):741-6.

95. Diamantis S, Waldorf HA. Rosacea: clinical presentation and pathophysiology. J Drugs Dermatol. 2006;5(1):8-12. 